\title{
THE MEANING OF INTRAGENOMIC CONFLICT
}

\author{
Andy Gardner ${ }^{1, *}$ and Francisco Úbeda ${ }^{2, *}$
}

1 School of Biology, University of St Andrews, St Andrews KY16 9TH, UK. 2 School of Biological Sciences, Royal Holloway University of London, Egham TW20 0EX, UK. * Correspondence: andy.gardner@st-andrews.ac.uk, f.ubeda@rhul.ac.uk

\begin{abstract}
Recent years have seen an explosion of interest in genes that function for their own good and to the detriment of other genes residing in the same genome. Such intragenomic conflicts are increasingly recognised to underpin maladaptation and disease. However, progress has been impeded by a lack of clear understanding as to what intragenomic conflict actually means, and an associated obscurity concerning its fundamental drivers. We develop a general theory of intragenomic conflict in which genes are viewed as inclusive-fitness-maximizing agents that come into conflict when their inclusive-fitness interests disagree. This yields a classification of all intragenomic conflicts into three categories according to whether genes disagree about where they have come from, where they are going, or where they currently are. We illustrate each of these three basic categories, survey and classify all known forms of intragenomic conflict, and discuss the implications for organismal maladaptation and human disease.
\end{abstract}

\section{INTRODUCTION}

Biological adaptation - the appearance of design in the living world - is conventionally viewed at the level of whole organisms, where it is understood to be driven by the action of natural selection and to function for the purpose of maximizing the individual's inclusive fitness [1-9]. But exploration of the molecular world of individual genes has uncovered many biological phenomena that cannot be explained with recourse to individual-level fitness and can only be interpreted according to the evolutionary interests of the genes themselves [10-14]. Such "intragenomic conflict" is increasingly recognised to underpin organismal maladaptation and human disease [13,15-22], with disorders ranging from autism to polycystic ovarian syndrome to mitochondrial disease all having been suggested to derive from conflicts of interest within the genome [23-28].

However, progress on this topic has been hampered by a lack of clear understanding as to what intragenomic conflict actually means. Although Burt \& Trivers [13] have assembled a comprehensive catalogue of examples that are broadly agreed to involve conflicts of interest within the genome, they offer no general definition, and the attempts of other researchers to define intragenomic conflict have turned out to be variously too restrictive - excluding some phenomena listed in Burt \& Trivers' catalogue - or too permissive - including instances of straightforward, individual-level adaptation (Table 1). This lack of basic understanding means that intragenomic conflict is often confused with other forms of evolutionary conflict - such as sexual conflict [29] - and that its fundamental drivers remain obscure.

Here, we resolve this problem by developing a general theory of intragenomic conflict from first principles, viewing genes as coming into conflict when their inclusive-fitness interests disagree. We comprehensively explore the ways in which genes may have inclusive-fitness disagreements, which leads to a classification of all intragenomic conflicts into three basic categories concerning when genes disagree as to where they have come from ("origin conflict"), where they are going ("destination conflict"), and where they currently are ("situation conflict"). We provide detailed illustrations of each of these categories, survey all known forms of intragenomic conflict - explaining where each fits into the general classification - and discuss its consequences for organismal maladaptation and human disease.

DEFINITION OF INTRAGENOMIC CONFLICT 
In general terms, conflicts of interest occur when different agents have different agendas, such that they disagree as to what is the best course of action. Accordingly, intragenomic conflict occurs when different genes residing in the same genome have different agendas. By "gene", we mean a physical scrap of nucleic acid [30], i.e. an arbitrary length, capable of some function. We do not mean "allele", i.e. the particular variant form exhibited by a gene, or "locus", i.e. the place where a gene resides [31]. And by "genome", we mean all of the genes carried by an individual organism whose combined phenotypic expression defines that organism.

A gene's agenda is to transmit copies of itself to future generations, via the reproduction of individual organisms. Individuals are valued in proportion to how well they transmit copies of the gene, and this is captured by the idea of "relatedness" [3, 32] (Box 1). Accordingly, the gene is favoured by natural selection to maximize the total reproductive success of its carrier and its carrier's social partners, each increment or decrement in reproductive success being weighted by the gene's relatedness to that individual. That is, the gene's agenda is to maximize its inclusive fitness [3, 30, 32] (Box 1).

Of course, scraps of nucleic acid do not have agendas in a literal sense. Rather, genes that achieve higher inclusive fitness tend to be represented by more descendant copies in future generations and, accordingly, the genes that accumulate in natural populations are expected to have the appearance of striving to maximize their inclusive fitness, in terms of the phenotypic effects that they have on the world [30]. Though unpalatable to some researchers [33-34], the analogy of agency is of great scientific utility as it facilitates prediction and the empirical testing of evolutionary theory and, consequently, underpins entire disciplines, such as behavioural ecology $[6-7,9,30]$.

The above ideas may be expressed mathematically (Box 1 ). If we hypothetically grant control of a phenotype to a particular gene $\mathbf{a}$, then the consequences of changing the phenotype in terms of this gene's inclusive fitness may be written as $\Delta H_{\mathrm{a}}=\sum_{j} \Delta \alpha_{\mathrm{aj}} r_{\mathrm{aj}}$, where $\Delta \alpha_{\mathrm{aj}}$ is the impact that this change has upon the reproductive success of the gene's carrier's $j$ th social partner (potentially including the gene's carrier herself, as well as individuals who don't currently exist but may do in the future) and $r_{\mathrm{aj}}$ is the relatedness valuation placed on that social partner by the focal gene [30] (Box 1). A gene that brings about this change in phenotype is favoured if $\Delta H_{\mathrm{a}}>0$. For example, if the change in phenotype involves an act of altruism by the carrier (social partner $j=0$, having $\Delta \alpha_{\mathrm{a} 0}=-c$ and $r_{\mathrm{a} 0}=1$ ) to a neighbour (social partner $j=1$, having $\Delta \alpha_{\mathrm{a} 1}=b$ and $\left.r_{\mathrm{a} 1}=r\right)$, then the phenotype is favoured if $-c+b r>0[3,30]$.

Alternatively, if we hypothetically grant control of the carrier's phenotype to a different gene b residing in the same genome, then the consequences of making the same phenotypic change in terms of this gene's inclusive fitness may be written as $\Delta H_{\mathbf{b}}=\sum_{j} \Delta \alpha_{\mathrm{b} j} r_{\mathrm{b} j}$, and it is favoured to bring about the change in phenotype if $\Delta H_{b}>0$ (Box 1). If both genes experience the same inclusive-fitness effect $\left(\Delta H_{\mathrm{a}}=\Delta H_{\mathrm{b}}\right)$ then they both agree as to whether to make the phenotypic change versus leave things as they were. However, if the genes experience different inclusive-fitness effects $\left(\Delta H_{\mathrm{a}} \neq \Delta H_{\mathrm{b}}\right)$ then they may find themselves in conflict with each other (Box 1). In particular, intragenomic conflict arises when the inclusive-fitness consequences of a particular phenotypic change are positive for one gene $\left(\Delta H_{a}>0\right)$ and negative for another gene $\left(\Delta H_{b}<0\right)$, such that they are favoured to pull the phenotype in different directions. That is, the ultimate source of intragenomic conflict lies in different genes residing in the same genome having different inclusive-fitness agendas.

\section{THREE KINDS OF INTRAGENOMIC CONFLICT}

How can different genes residing within the same genome have different inclusive-fitness agendas? Consideration of the components of inclusive fitness indicates three ways in which this may occur. Here, we describe these three kinds of intragenomic conflict and provide an illustration of each involving disagreement between a male's autosomal versus his X-linked genes, though we emphasise that this scheme applies much more generally. These three kinds of intragenomic conflict are readily distinguished from each other, but in particular scenarios two or more may be in operation simultaneously. 
Origin conflict - One possibility is that genes disagree as to their relatedness valuations of their carrier's nondescendant relatives $\left(r_{\mathrm{aj}} \neq r_{\mathrm{b} j}\right.$, where social partner $j$ is a nondescendant relative; Box 1). As nondescendant relatives are individuals who share a common ancestry, then this disagreement occurs when the genes disagree as to where they have come from. We term this "origin conflict".

An example of origin conflict is that arising between a male's autosomal genes versus his Xlinked genes on account of the former being equally likely to originate from either of his parents and the latter definitely originating from his mother, such that these genes may make different relatedness valuations of his maternal and paternal relations. For instance, if the male may enact altruism towards his maternal siblings, and if female promiscuity means that these need not be his paternal siblings, then he will find his X-linked genes relatively more inclined, and his autosomal genes relatively less inclined, to such altruism (Box 2).

Destination conflict - Alternatively, genes may disagree as to their relatedness valuations of their carrier's descendants $\left(r_{\mathrm{aj}} \neq r_{\mathrm{b}}\right.$, where social partner $j$ is a descendant; Box 1). Specifically, if two genes disagree as to their probability of being transmitted to particular descendants then they may consequently disagree as to their relatedness to these descendants. We term this "destination conflict".

An example of destination conflict is that arising between a male's autosomal genes versus his X-linked genes on account of the former being equally likely to transmit to his daughters and sons and the latter being transmitted only to his daughters, such that these genes may make different relatedness valuations of his daughters and sons. For instance, if the male may enact paternal care towards his daughters, then he will find his X-linked genes relatively more inclined, and his autosomal genes relatively less inclined, to such paternal care (Box 3 ).

Situation conflict - Finally, genes may disagree as to the consequences that a phenotypic change will have for their carrier's and/or other social partners' reproductive success $\left(\Delta \alpha_{\mathrm{aj}} \neq\right.$ $\Delta \alpha_{b j}$; Box 1). Since the fitness of an individual organism is an objective fact that is determined by context, the genes may have different expectations as to the fitness consequences of their actions when they disagree as to their carrier's context. We term this "situation conflict".

An example of situation conflict is that arising between a male's autosomal genes versus his $\mathrm{X}$-linked genes on account of $\mathrm{X}$-linked genes being relatively more concentrated in females, such that - in the absence of other information - they attach greater likelihood to their carrier being female. For instance, if the male may exhibit sexually-attractive ornamentation that incurs a mortality cost irrespective of its bearer's sex and yields a mating advantage when its bearer is male, then his X-linked genes will be relatively less inclined, and his autosomal genes relatively more inclined, to have him exhibit such ornamentation, the former genes having lower confidence in their carrier being male (Box 4).

\section{CLASSIFICATION OF INTRAGENOMIC CONFLICTS}

Identification of these three kinds of intragenomic conflict enables a general classification that encompasses all known examples that have been definitively catalogued by Burt \& Trivers [13] (Figure 1). Here, we show where each example of intragenomic conflict fits into the general classification, with a particular focus on three paradigmatic examples.

The first paradigmatic example of intragenomic conflict occurs between an individual's maternal-origin versus paternal-origin autosomal genes [35-36]. According to the kinship theory of genomic imprinting, this conflict drives the evolution of parent-of-origin specific gene expression [37]. For example, if an individual may enact altruism towards maternal siblings that need not to be paternal siblings, then any autosomal genes that know themselves to be of maternal origin will be relatively more inclined, and any autosomal genes that know themselves to be of paternal origin will be relatively less inclined, to such altruism (see Supplementary Material). According to our classification, this is an example of origin conflict (Figure 1). 
Other examples of origin conflict are: conflicts between genes residing in cytoplasmic organelles and genes residing in the nucleus on account of the former having an exclusivelymaternal origin in most animals and the latter having maternal or paternal origin with equal probability; and conflict between genes residing on $\mathrm{X}$ chromosomes, genes residing on $\mathrm{Y}$ chromosomes and genes residing on autosomes, on account of a maternal origin being more likely than a paternal origin for X-linked genes, less likely for $\mathrm{Y}$-linked genes, and equally likely for autosomal genes (Box 2; Figure 1).

The second paradigmatic example of intragenomic conflict occurs between meiotic driver genes versus non-driver autosomal genes, which is understood to be responsible for the evolution of complex chromosomal architectures [38-41]. Specifically, meiotic drive results in the majority of the carrier's offspring receiving a copy of the driving gene and a minority of the offspring receiving a copy of the non-driving homologue, and may be favoured by the driving gene as it is more related to the resulting offspring and disfavoured by the non-driving homologue as it is less related to these offspring (see Supplementary Material). Accordingly, this is an instance of destination conflict (Figure 1).

Other instances of destination conflict are: conflicts between genes causing maternal or paternal genome elimination (biased gene conversion, homing endonucleases) and their homologues that do not on account of the former being present in more than their fair share of offspring and the latter in less than their fair share; conflicts between transposable elements and their homologues that do not transpose on account of the former having more copies in the genome of offspring than the latter; conflict between genes residing in cytoplasmic organelles and genes residing in the nucleus, for example on account of the former being passed on only by a female carrier's daughters and the latter being passed on by her sons and daughters; conflict between genes residing in sex chromosomes (X or $\mathrm{Y})$ and genes residing in autosomes on account of, for example, a male passing on his $X$-linked genes only to his daughters, his Y-linked genes only to his sons, and his autosomal genes to offspring of both sexes (Box 3; Figure 1).

The third paradigmatic example of intragenomic conflict occurs between greenbeard genes and genes residing elsewhere in the genome. Specifically, a greenbeard gene is one that encodes a phenotypic marker (such as a green beard) and also a tendency to behave preferentially towards social partners exhibiting this marker (such as altruism towards greenbearded neighbours) [3, 42-43]. Whether and how greenbeard genes may be embroiled in intragenomic conflict has long been debated [43-47]: most recently, Biernaskie et al. [46] have shown that different genes exerting control over a greenbeard phenotype may come into conflict, but the reasons for this have remained obscure. Framing such greenbeard effects in terms of the present inclusive-fitness approach, we find that this conflict emerges as a consequence of different genes attaching different likelihoods to social partners exhibiting the greenbeard phenotype, i.e. disagreement as to their carrier's social context (see Supplementary Material). Accordingly, this is an instance of situation conflict (Figure 1).

Situation conflicts may be relatively common in scenarios where different genes are differently associated with different classes of carrier. Above, we considered a scenario in which autosomal versus $\mathrm{X}$-linked genes attach different likelihoods to their carrier being male versus female, and hence expect different fitness consequences from expressing sexually-attractive ornamentation (Box 4; Figure 1). Analogous conflicts may also extend to genes residing on $Y$ chromsomes, that are certain their carrier is male (Figure 1). Situation conflicts could also occur in previously-hypothesised scenarios in which genomic cues indicate aspects of the local environment - such as homozygosity indicators of local inbreeding [48] or the presence of a locally-adapted allele providing information about environmental context [49-50] - if these are differentially accessible to different genes.

\section{DISCUSSION}

We have developed a general theory of intragenomic conflict, taking an inclusive-fitness approach to characterise the interests of genes and providing a clear definition of genetic conflict in terms of a mismatch between these interests. By breaking down inclusive fitness into its component parts, we have shown that intragenomic conflicts arise when different 
genes residing in the same genome disagree as to the fitness consequences of phenotypic decisions and / or their relatedness to their carrier's social partners. More proximately, we have shown that such differences may arise as a consequence of genes having different information concerning their origin, destination or current situation. Our treatment provides the first formal framework that captures all forms of intragenomic conflict within a single, unified and comprehensive scheme within which the biology of intragenomic conflicts may be studied, conceptualised and clearly communicated.

Many previous definitions of intragenomic conflict have been too restrictive to capture all its different forms. In particular, Cosmides and Tooby [51] and Werren [52] have taken a transmission-focused approach that sees intragenomic conflict arising as a consequence of different genes having different modes of transmission such that, for example, maternallytransmitted cytoplasmic genes may come into conflict with mendelian-transmitted autosomal genes but there can be no intragenomic conflict between two mendelian-transmitted autosomal genes. In contrast, our framework imposes no such restriction, and indeed highlights that there is abundant scope for intragenomic conflict between genes that share the same mode of transmission, such as between mendelian-transmitted autosomal genes of different parental origin. Similarly restrictive is Grafen's [5] treatment of intragenomic conflict which, based on the assumption of fair mendelian inheritance, excludes many intragenomic conflicts involving genes exhibiting non-mendelian transmission, such as meiotic drivers. Our framework avoids being too restrictive by focusing directly upon genes' inclusive-fitness agendas and determining when these agendas diverge.

Conversely, some previous definitions of intragenomic conflict have been too permissive, inadvertently diagnosing genetic conflicts where none exist. Hurst et al. [33] have defined intragenomic conflict in terms of the spread of one gene creating the context for the spread of another that has the opposite phenotypic effect, with both genes being expressed in the same individual, and this definition has been very widely taken up in the evolutionary literature. However, Biernaskie et al. [46] have pointed out that this definition may incorrectly diagnose classical organismal fine-tuning of adaptation as intragenomic conflict. For example, in a population in which average body size is below the optimum, a mutation that increases body size may be favoured even if it slightly overshoots the optimum, such that mutations arising at other loci and expressed in the very same individuals will be favoured to pull the phenotype back in the opposite direction [46, 53]. Again, our framework avoids being too permissive by engaging directly with genes' inclusive-fitness agendas and determining when these actually differ.

Intragenomic conflicts have often been viewed through the prism of multilevel selection, an approach to social evolution that separates the dynamics of selection acting within versus between individual organisms and other levels of biological organisation [52, 54-56]. In particular, some proponents of this view have taken intragenomic conflict to be synonymous with a gene being selectively favoured at a within-individual level yet selectively disfavoured at a between-individual level [52, 54-56]. However this, too, provides an inadequate framework for capturing all forms of intragenomic conflict, as it excludes all those instances in which genes exhibit fair mendelian transmission. For example, conflicts of interest between maternal-origin versus paternal-origin genes in the context of social partners being differentially related via their mothers and their fathers need not be driven by within-individual selection but rather by between-individual kin selection, with the genes simply disagreeing as to the relatedness valuation of social partners [18-20, 35, 57-58].

Our inclusive-fitness framework departs from previous ideas about gene-level adaptation in terms of which biological entities we are considering to be adaptive agents. Whereas Dawkins [42] defines the "selfish gene" as a distributed agent that comprises every copy of a particular allele in an evolving lineage (see also [59-60]), we define the gene as a single, physical scrap of nucleic acid (see also [30]). This is crucial if we are to consider conflicts of interest between, for instance, maternal-origin genes and paternal-origin genes, as it is only the physical genic token - and not the allelic type - that has a parent of origin [58]. A consequence of our definition is that - just like whole organisms - inclusive-fitnessmaximizing genes may behave altruistically, spitefully and mutually-beneficially, rather than purely selfishly [30]. Similarly, we have defined the genome as the physical aggregate of all 
genes carried by an organism whose combined expression defines that organism's phenotype. That is, it is a material object associated with a particular individual, rather than an informational blueprint for an entire species or an evolving lineage's genepool [61]. The latter sense would lead to intragenomic conflict encompassing all conflicts arising between genes residing in the same gene pool - even those residing in different bodies - including conflicts between mates [62], parents and offspring [63] and siblings [64]. However, broad consensus holds that intragenomic conflict should not cover all these phenomena [13].

Intragenomic conflict has often been considered in conjunction with sexual conflict under the generalised heading of "genetic conflict" [52-53, 65]. Indeed, some researchers have even suggested that sexual conflict is a form of intragenomic conflict [29]. Our inclusive-fitness framework clarifies the connections and crucial differences between these evolutionary phenomena. First, so-called "interlocus sexual conflict" refers to antagonistic interaction between a male and a female whereby a gene expressed in one of these individuals leads to a fitness increase for its carrier and a fitness decrease for the other individual, potentially providing the context for selection to favour a gene in the other individual that induces the opposite effect [66-67]. Whilst this is true conflict, involving a divergence of male and female optima, it is not intragenomic conflict because the genes involved reside in different individuals. Second, so-called "intralocus sexual conflict" refers to instances where a gene induces a phenotypic effect that is beneficial when the gene resides in a male but deleterious when it resides in a female, or vice versa [66-67]. This is not true conflict, but rather a tension experienced by a single gene having to balance opposing selection pressures. However, if two genes residing in the same individual have different information regarding their carrier's sex, then they may disagree as to how these pressures balance out, i.e. intragenomic situation conflict of the kind investigated in Box 4.

Intragenomic conflict is believed to be an important driver of organismal maladaptation and associated disease. Although the link between these phenomena has received some attention in relation to particular examples, it is impossible to achieve a general understanding of how intragenomic conflict drives maladaptation without first having a general understanding of intragenomic conflict itself. Having provided a general definition of intragenomic conflict, a comprehensive theory of its evolutionary drivers, and an exhaustive classification of all its forms, we suggest that a general understanding of the resulting maladaptation is now possible and that this requires urgent attention. Whilst we have taken a standard "battleground" [68] approach that identifies conflict by hypothetically assigning full control of the contentious phenotype to each gene in turn in order to assess that gene's preferences, an explicit model of shared control is necessary for exploring the "resolution" [68] of intragenomic conflict and resulting maladaptation. However, one immediate avenue for applying our framework concerns the identification of genomic "hotspots" for maladaptation: for example, our illustrative analyses have underlined that whilst the unimprinted, mendelian-inherited autosomal genes that make up the bulk of the genome have relatively little scope for coming into conflict with each other, X-linked genes may be simultaneously embroiled in multiple origin, destination and situation conflicts with the rest of the genome. By identifying the fundamental drivers of intragenomic conflicts, we are better equipped to locate them.

Moreover, whilst the link between intragenomic conflict and maladaptation has generally been regarded as straightforward and hence not requiring further fundamental investigation, our inclusive-fitness analysis reveals that the link is more complicated and requires renewed attention. For example, the multilevel-selection approach has actually defined genomic outlaws in terms of their incurring a loss of fitness for the organism [54], giving the impression that maladaptation is a trivial consequence of intragenomic conflict. In contrast, inclusivefitness conflicts between, say, an individual's maternal-origin versus paternal-origin genes need not obviously lead to organismal maladaptation, as an averaging over these genes' divergent interests exactly recovers the individual's inclusive-fitness optimum [10,69]. Instead, maladaptation may arise in such scenarios when the conflict is resolved in favour of one gene and against the other, as predicted by the "loudest-voice prevails" principle that involves one gene at an imprinted locus winning the conflict, such that the phenotype is perturbed away from the individual's optimum [10-11, 70-71]. In addition, conflict between imprinted loci has been implicated in driving an escalation in the expression of genes with antagonistic phenotypic effects that may incur significant costs to the individual [72]. The 
resulting tension is understood to render the individual less robust to mutational perturbation, such that deleterious mutations occurring at conflicted loci are expected to have larger phenotypic effects than those occurring at other loci, as exemplified by Prader-Willi syndrome $[15,75]$. It is remarkable that inclusive-fitness theory, which was developed to explain and characterize the adaptations of whole organisms, provides - in its gene-level formulation - a predictive and explanatory framework for understanding patterns of organismal maladaptation and human disease.

\section{CORRESPONDENCE}

Correspondence should be addressed to Andy Gardner and/or Francisco Úbeda.

\section{ACKNOWLEDGMENTS}

We thank Steven Frank, Michael Morrissey and three anonymous reviewers for helpful comments and discussion, and Francisco Úbeda Izargain for an original source of intragenomic conflict. AG is supported by an Independent Research Fellowship from the Natural Environment Research Council (NE/K009524/1).

\section{AUTHOR CONTRIBUTIONS}

AG and FÚ conceived the study, performed the analyses and wrote the paper.

\section{COMPETING INTERESTS}

The authors declare no competing financial interests.

\section{REFERENCES}

1. Darwin, C.R. The origin of species (John Murray,1859).

2. Fisher, R.A. The genetical theory of natural selection (Clarendon,1930).

3. Hamilton, W.D. The genetical evolution of social behaviour. J. Theor. Biol. 7, 1-52 (1964).

4. Price, G.R. Selection and covariance. Nature 227, 520-521 (1970).

5. Grafen, A. Optimisation of inclusive fitness. J. Theor. Biol. 238, 541-563 (2006).

6. Gardner, A. Adaptation as organism design. Biol. Lett. 6, 861-864 (2009).

7. West, S.A. \& Gardner, A. Adaptation and inclusive fitness. Curr. Biol. 23, R577-R584 (2013).

8. Grafen, A. Formal Darwinism, the individual-as-maximising-agent analogy, and bethedging. Proc. R. Soc. Lond. B 266, 799-803 (1999).

9. Davies, N.B., Krebs, J.R. \& West, S.A. An introduction to behavioural ecology. (WileyBlackwell, 2012).

10. Haig, D. Genomic imprinting and kinship. (Rutgers, 2002).

11. Úbeda, F. \& Haig, D. Sex-specific meiotic drive and selection at an imprinted locus. Genetics 167, 2083-2095 (2004).

12. Úbeda, F. \& Normark, B.B. Male killers and the origins of paternal genome elimination. Theor. Pop. Biol. 70, 511-526 (2006).

13. Burt, A. \& Trivers, R. Genes in conflict: the biology of selfish genetic elements (Harvard, 2006).

14. Foster, K.R. The sociobiology of molecular systems. Nat. Rev. Genet. 12, 193-203 (2011).

15. Úbeda, F. \& Wilkins, J.F. Imprinted genes and human disease, an evolutionary perspective. Adv. Exp. Med. Biol. 626, 101-115 (2008).

16. Summers, K., da Silva, J. \& Farwell, M.A. Intragenomic conflict and cancer. Med. Hypoth. 59, 170-179 (2002).

17. Constancia, M., Kelsey, G. \& Reik, W. Resourceful imprinting. Nature 432, 53-57 (2004).

18. Úbeda, F. \& Gardner, A. A model for genomic imprinting in the social brain: juveniles.

Evolution 64, 2587-2600 (2010).

19. Úbeda, F.\& Gardner, A. A model for genomic imprinting in the social brain: adults. Evolution 65, 462-475 (2011).

20. Úbeda, F. \& Gardner, A. A model for genomic imprinting in the social brain: elders.

Evolution 66, 1567-1581 (2012). 
21. Crespi, B., Foster, K. \& Úbeda, F. First principles of Hamiltonian medicine. Phil. Trans. R. Soc. B 369, 20130366 (2014).

22. Haig, D. Maternal-fetal conflict, genomic imprinting and mammalian vulnerabilities to cancer. Phil. Trans. R. Soc. B 370, 20140178 (2015).

23. Hague, W.M., Adams, J., Reeders, S.T., Peto, T.E. \& Jacobs, H.S. Familial polycystic ovaries: a genetic disease? Clin. Endocrinol. 29, 593-605 (1988).

24. Haig, D. \& Wharton, R. Prader-Willi syndrome and the evolution of human childhood. Am. J. Hum. Biol. 15, 320-329 (2003).

25. Úbeda, F. Evolution of genomic imprinting with biparental care: implications for Prader-

Willi and Angelman syndromes. PLoS Biol. 6, 1678-1692 (2008).

26. Crespi, B. \& Badcock, C. Psychosis and autism as diametrical disorders of the social brain. Behav. Brain. Sci. 31, 241-320 (2008).

27. Belancio, V.P., Deininger, P.L. \& Roy-Engel, A.M. LINE dancing in the human genome: transposable elements and disease. Genom. Med. 1, 97 (2009).

28. Chou, J.Y. \& Leu, J.Y. The red queen in mitochondria: cyto-nuclear co-evolution, hybrid breakdown and human disease. Front. Genet. 6, 1-8 (2015).

29. Gavrilets, S. Is sexual conflict an engine of speciation? Cold Spring Harb. Perspect. Biol. 6, a017723 (2014).

30. Gardner, A. \& Welch, J.J. A formal theory of the selfish gene. J. Evol. Biol. 24, 1801-1813 (2011).

31. Rousset, F. Genetic structure and selection in subdivided populations. (Princeton, 2004).

32. Hamilton, W.D. Altruism and related phenomena, mainly in social insects. Ann. Rev. Ecol. Syst. 3, 193-232 (1972).

33. Hurst, L.D., Atlan, A. \& Bengtsson, B.O. Genetic conflicts. Q. Rev. Biol. 71, 317-364 (1996).

34. Charlesworth, B. Conflicts of interest. Curr. Biol. 16, R1009-R1011 (2006).

35. Haig, D. Parental antagonism, relatedness asymmetries, and genomic imprinting. Proc. Biol. Sci. 264, 1657-1662 (1997).

36. Úbeda, F. \& Haig, D. Dividing the child. Genetica 117, 103-110 (2003).

37. Moore, T. \& Haig, D. Genomic imprinting in mammalian development: a parental tug-ofwar. Trends Genet. 7, 45-49 (1991).

38. Crow, J.F. The ultraselfish gene. Genetics 118, 389-391 (1988).

39. Lindholm, A.K. et al. The ecology and evolutionary dynamics of meiotic drive. Trends Ecol. Evol. 31, 315-326 (2016).

40. Larracuente, A.M. \& Presgraves, D.C. The selfish Segregation Distorter gene complex of Drosophila melanogaster. Genetics 192, 33-53 (2012).

41. Leigh, E.G. How does selection reconcile individual advantage with the good of the group? Proc. Natl. Acad. Sci. USA 74, $4542-4546$ (1977).

42. Dawkins, R. The selfish gene (Oxford,1976).

43. Gardner, A. \&West, S.A. Greenbeards. Evolution 64, 25-38 (2010).

44. Ridley, M. \& Grafen, A. Are greenbeard genes outlaws? Anim. Behav. 29, 954-955 (1981)

45. Dawkins, R. The extended phenotype (Oxford,1982).

46. Biernaskie, J.M., West, S.A. \&Gardner, A. Are greenbeards intragenomic outlaws?

Evolution 65, 2729-2742 (2011).

47. Alexander, R.D. \& Borgia, G. Group selection, altruism, and levels of organization of life. Ann. Rev. Ecol. Evol. Syst. 9, 449-474 (1978).

48. Seger, J. Evolution of responses to relative homozygosity. Nature 262, 579-580 (1976).

49. Dall, S.R.X., McNamara, J.M. \& Leimar, O. Genes as cues: phenotypic integration of

genetic and epigenetic information from a Darwinian perspective. Trends Ecol. Evol. 30, 327333 (2015).

50. Leimar, O., Dall, S.R.X., Hammerstein, P. \& McNamara, J.M. Genes as cues of relatedness and social evolution in heterogeneous environments. PLoS Comput. Biol. 12, e1005006 (2016).

51. Cosmides, L.M. \& Tooby, J. Cytoplasmic inheritance and intragenomic conflict. J. Theor. Biol. 89, 83-129 (1981).

52. Werren, J.H. Selfish genetic elements, genetic conflict, and evolutionary innovation. Proc. Natl. Acad. Sci. USA 108, 10863-10870 (2011).

53. Rice, W.R. Nothing in genetics makes sense except in the light of genomic conflict. Ann. Rev. Ecol. Evol. Syst. 44, 217-237 (2013).

54. Okasha, S. Evolution and the levels of selection (Oxford, 2006). 
55. Pomiankowski, A. in Levels of selection in evolution (ed Keller, L.) 121-152 (Princeton, 1999).

56. Spencer, H.G. Intragenomic conflict. eLS (2003).

57. Brandvain, Y. Matrisibs, patrisibs, and the evolution of imprinting on autosomes and sex chromosomes. Am. Nat. 176, 511-521 (2010).

58. Gardner, A. Genomic imprinting and the units of adaptation. Heredity 113, 104-111

(2014).

59. Williams, G.C. Adaptation and natural selection. (Princeton, 1966).

60. Williams, G.C. Natural selection: domains, levels, and challenges. (Oxford, 1992).

61. Stencel, A. \& Crespi, B. What is a genome? Molec. Ecol. 22, 3437-3443 (2013).

62. Trivers, R.L. in sexual selection and the descent of man 1871-1971. (ed Campbell, B.)

136-179 (Aldine, 1972).

63. Trivers, R.L. Parent-offspring conflict. Am. Zool. 14, 249-264 (1974).

64. Mock, D.W. \& Parker, G.A. The evolution of sibling rivalry (Oxford, 1997).

65. Rice, W.R. \& Holland, B. The enemies within: intergenomic conflict, interlocus contest evolution (ICE), and the intraspecific Red Queen. Behav. Ecol. Sociobiol. 41, 1-10 (1997).

66. Chapman, T., Arnqvist, G., Bangham, J. \& Rowe, L. Sexual conflict. Trends Ecol. Evol. 18, 41-47 (2003).

67. Haig, D., Úbeda, F. \& Patten, M.M. Specialists and generalists: the sexual ecology of the genome. Cold Spring Harb. Perspect. Biol. 6, 017525 (2014).

68. Godfray, H.C.J. Evolutionary theory of parent-offspring conflict. Nature 376, 133-138

(1995).

69. Gardner, A. \& Ross, L. Mating ecology explains patterns of genome elimination. Ecol. Lett. 17, 1602-1612 (2014).

70. Haig, D. Placental hormones, genomic imprinting, and maternal-fetal communication. J. Evol. Biol. 9, 356-380 (1996).

71. Farrell, E. J., Úbeda, F. \& Gardner, A. Intragenomic conflict over dispersal. Am. Nat. 186, E61-E71 (2015).

72. Wilkins, J.F. Genomic imprinting and conflict-induced decanalization. Evolution 65, $537-$ 5532011.

73. Bulmer, M.G. Theoretical evolutionary ecology (Sinauer, 1994).

74. Gardner, A. The genetical theory of multilevel selection. J. Evol. Biol. 28, 305-319 (2015). 75. Úbeda, F. \& Gardner, A. Mother and offspring in conflict: why not? PLoS Biology 13, e1002084 (2015) 


\section{Box 1 | Mathematics of intragenomic conflict}

Let $\mathbf{a}$ be a focal genic actor, and $j \in J$ be the social partners of this gene's carrier, with $j=0$ representing the gene's carrier herself. Let $G_{j}$ be the set of all genes in individual $j$ 's genome. Let $s_{\mathbf{a}} \in S$ be gene a's strategy and $\Pi_{0} \in \Pi$ be the phenotype of gene a's carrier.

Gene a's inclusive fitness is:

$H_{\mathbf{a}}\left(\pi_{0}\left(s_{\mathbf{a}}\right)\right)=\sum_{j \in J} \alpha_{\mathbf{a} j}\left(\pi_{0}\left(s_{\mathbf{a}}\right)\right) \frac{\sum_{g \in G_{j}} v_{\mathbf{a} j g} p_{\mathbf{a} j g}}{\sum_{g \in G_{0}} v_{\mathbf{a} 0 g} p_{\mathbf{a} 0 g}}$

where $\alpha_{a j}$ is gene a's estimate of the additive impact upon the fitness of social partner $j$ arising from gene $a$ 's carrier exhibiting phenotype $\pi_{0}, v_{\text {aig }}$ is gene $a$ 's estimate of the reproductive value of gene $g$ in social partner $j$ [73], and $p_{\text {ajg }}$ is gene a's estimate of its consanguinity (i.e. probability of identity by descent [73]) to gene $g$ in social partner $j$ (see Supplementary Material for derivation). Note that reproductive value is calculated under the assumption of neutrality: this is appropriate because, although actual genetic contributions to the future will be modulated by selection acting in future generations, such effects should not be conflated with selection acting in the present generation [74]. This ratio of reproductive-value weighted consanguinity is the life-for-life formulation of the kin selection coefficient of relatedness [32].

Gene a's agenda is to maximise its own inclusive fitness:

$\max _{s_{\mathbf{a}} \in S} H_{\mathbf{a}}\left(\pi_{0}\left(s_{\mathbf{a}}\right)\right)$

The population frequency of identical-by-descent copies of gene a increases when the inclusive fitness effect $\partial H_{a} / \partial s_{a}$ is positive:

$\underbrace{\frac{\partial \mathcal{H}_{\mathrm{a}}}{\partial S_{\mathrm{a}}}}_{\Delta \mathcal{H}_{\mathrm{a}}}=\sum_{j \in J} \underbrace{\frac{\partial \alpha_{\mathrm{a} j}}{\partial \pi_{0}}}_{\Delta \alpha_{\mathrm{a} j}} \frac{\partial \pi_{0}}{\partial s_{\mathrm{a}}} \frac{\underbrace{\sum_{g \in G_{j}} v_{\mathrm{a} j g} p_{\mathrm{a} j g}}_{r_{\mathrm{a} j}}}{\sum_{g \in G_{0} v_{\mathrm{a} o g} p_{\mathrm{a} 0} g}}$

Notice that if there is no consanguinity between loci (e.g. no transposition) and if there is no covariance within loci between consanguinity and reproductive value (e.g. no paternal genome elimination with higher consanguinity via matrilines $[13,69])$, then:

$r_{\mathrm{a} j}=\frac{v_{\mathrm{a} j} p_{\mathrm{a} j}}{v_{\mathrm{a} 0} p_{\mathrm{a} 0}}$

where $v_{\mathrm{aj}}$ is gene a's estimate of the total reproductive value of social partner $j$ 's genes residing at the same locus and $p_{\mathrm{aj}}$ is gene a's estimate of its consanguinity to a random gene drawn from the same locus from social partner $j$.

Hypothetically granting gene a full control of its carrier's phenotype $\pi_{0}-$ that is, $\pi_{0}=s_{a}-$ gene a's agenda is to set its carrier's phenotype to that which maximizes its own inclusive fitness:

$\max _{\pi_{0} \in \Pi} H_{\mathbf{a}}\left(\pi_{0}\right)$

The optimal phenotype from the perspective of genic actor $\mathbf{a}$ is $\pi_{0}^{* \mathbf{a}}$, which satisfies:

$H_{\mathbf{a}}\left(\pi_{0}^{* \mathbf{a}}\right) \geq H_{\mathbf{a}}\left(\pi_{0}\right) \quad \forall \pi_{0} \in \Pi$

Conversely, hypothetically granting full control of the carrier's phenotype to a different gene $\mathbf{b}$ residing in the same genome - that is, $\Pi_{0}=s_{\mathbf{b}}$ - gene b's agenda is given by:

$\max _{\pi_{0} \in \Pi} H_{\mathbf{b}}\left(\pi_{0}\right)$

The optimal phenotype from the perspective of genic actor $\mathbf{b}$ is $\pi_{0}^{* \mathbf{b}}$, which satisfies: 
$H_{\mathbf{b}}\left(\pi_{0}^{* \mathbf{b}}\right) \geq H_{\mathbf{b}}\left(\pi_{0}\right) \quad \forall \pi_{0} \in \Pi$

Accordingly, there is intragenomic conflict between genes $\mathbf{a}$ and $\mathbf{b}$ when:

$\pi_{0}^{* \mathbf{a}} \neq \pi_{0}^{* \mathbf{b}}$

A necessary (although not sufficient) condition for the existence of intragenomic conflict is that the inclusive fitness effect differs between genic actors, that is:

$\Delta H_{\mathbf{a}} \neq \Delta H_{\mathbf{b}}$

This is achieved either when the genes differ in their estimated relatedness to some or all social partners:

$\exists j \in J$ such that $r_{\mathbf{a} j} \neq r_{\mathbf{b} j}$

or the genes differ in their estimates as to the fitness consequences of a phenotypic change:

$\exists j \in J$ such that $\frac{\partial \alpha_{\mathrm{a} j}}{\partial \pi_{0}} \neq \frac{\partial \alpha_{\mathrm{b} j}}{\partial \pi_{0}}$ 


\section{Box 2 | Origin conflict}

As an illustration of intragenomic conflict of the origin type, consider a scenario in which an actor gene a residing in a male causes him to undertake an act of altruism towards his maternal siblings, providing a fitness benefit $B$ to them whilst reducing his own fitness by $C$, in the context of a large, randomly-mating population in which females are highly promiscuous. The gene's inclusive fitness is increased by this act of altruism if $-c_{\mathrm{a}}+b_{\mathrm{a}} r_{\mathrm{a} \text {, sibling }}>0$, where $c_{\mathrm{a}}$ $=C, b_{\mathrm{a}}=B$ and $r_{\mathrm{a}, \text { sibling }}$ is the relatedness of gene $\mathbf{a}$ to the male's maternal siblings.

Taking the perspective of an autosomal gene $\mathbf{A}$, its relatedness to its male carrier's maternal siblings is $r_{\mathrm{A} \text {, sibling }}=1 / 2 \times 1 / 2+1 / 2 \times 0=1 / 4$, because: with probability $1 / 2$ the gene originated from the male's mother, in which case it is related by $1 / 2$ to the male's maternal siblings; and with probability $1 / 2$ the gene originated from the male's father, in which case it is unrelated to the male's maternal siblings (see Supplementary Material). Accordingly, the autosomal gene A favours the act of altruism if $C / B<1 / 4$. Alternatively, taking the perspective of an X-linked gene $X$, its relatedness to maternal siblings is $r_{\mathrm{X}, \text { sibling }}=1 \times 1 / 2=1 / 2$, because: with probability 1 the gene originated from the male's mother, and hence is related by $1 / 2$ to the male's siblings (see Supp Mat). Accordingly, the $X$-linked gene $X$ favours the act of altruism if $C / B<1 / 2$.

This difference in the relatedness valuations made by the autosomal versus $X$-linked genes may lead to an intragenomic conflict of interest with respect to the altruism phenotype, depending upon the ratio of fitness cost and benefit: if the cost is relatively small $(C / B<1 / 4)$, then both autosomal and X-linked genes favour altruism (no conflict); if the cost is relatively large $(C / B>1 / 2)$, then neither gene favours altruism (no conflict); and if the cost is intermediate $1 / 4<C / B<1 / 2$ ), then the autosomal gene disfavours altruism and the X-linked gene favours altruism (intragenomic conflict).

<Figure B2 here> 


\section{Box 3 | Destination conflict}

As an illustration of intragenomic conflict of the destination type, consider a scenario in which an actor gene a residing in a male causes him to undertake an act of paternal care towards his daughters, providing a fitness benefit $B$ to them whilst reducing his own fitness by $C$, in the context of a large, randomly-mating population with an even sex ratio. The gene's inclusive fitness is increased by this act of altruism if $-c_{\mathrm{a}}+b_{\mathrm{a}} r_{\mathrm{a} \text {,daughter }}>0$, where $c_{\mathrm{a}}=C, b_{\mathrm{a}}=$ $B$ and $r_{\mathbf{a} \text {,daughter }}$ is the relatedness of gene $\mathbf{a}$ to the male's daughters.

Taking the perspective of an autosomal gene $\mathbf{A}$, its relatedness to the male's daughters is $r_{\mathrm{A} \text {,daughter }}=1 / 2$, because with probability $1 / 2$ it is passed onto each daughter (see Supp Mat) and, accordingly, the autosomal gene $A$ favours the act of paternal care if $C / B<1 / 2$. Alternatively, taking the perspective of an X-linked gene $\mathbf{X}$, its relatedness to the male's daughters is $r_{\mathrm{X} \text {,daughter }}=1$, because with probability 1 it is passed onto each daughter (see Supplementary Material) and, accordingly, the $X$-linked gene $\mathbf{X}$ favours the act of paternal care if $C / B<1$.

This difference in the relatedness valuations made by the autosomal versus $\mathrm{X}$-linked genes may lead to an intragenomic conflict of interest with respect to the paternal care phenotype, depending upon the ratio of fitness cost and benefit: if the cost is relatively small $(C / B<1 / 2)$, then both autosomal and X-linked genes favour paternal care (no conflict); if the cost is relatively large $(C / B>1)$, then neither gene favours paternal care (no conflict); and if the cost is intermediate $1 / 2<C / B<1$ ), then the autosomal gene disfavours paternal care and the $X$ linked gene favours paternal care (intragenomic conflict).

$<$ Figure B3 here> 


\section{Box 4 | Situation conflict}

As an illustration of intragenomic conflict of the situation type, consider a scenario in which an actor gene a causes its carrier to exhibit a sexually-selected ornament that incurs a fitness cost $C$ - irrespective of the individual's sex - on account of increased attention to predators, and yields a fitness benefit $B$ - for males only - on account of increased mating success, in the context of a large, randomly-mating population with an even sex ratio. The gene's inclusive fitness is increased by exhibiting the ornament if $-c_{\mathrm{a}}>0$, where $c_{\mathrm{a}}=-C+m B$ and $m$ is the relative likelihood that the gene's carrier is male (note that the indirect component of inclusive fitness is zero because this the ornament has no impact on the fitness of relatives).

Taking the perspective of an autosomal gene $\mathbf{A}$, the relative likelihood that its carrier is male is $1 / 2$, as autosomal genes occur equally frequently in males and females (see Supplementary Material) and, accordingly, the autosomal gene $A$ favours exhibiting the ornament if $C / B<1 / 2$. Alternatively, taking the perspective of an $\mathbf{X}$-linked gene $\mathbf{X}$, the relative likelihood that its carrier is male is $1 / 3$, as X-linked genes occur twice as frequently in females as they do in males (see Supp Mat) and, accordingly, the $\mathbf{X}$-linked gene $\mathbf{X}$ favours exhibiting the ornament if $C / B<1 / 3$.

This difference in the perceived likelihood of residing in a male versus female made by the autosomal versus $X$-linked genes may lead to an intragenomic conflict of interest with respect to the ornament phenotype, depending upon the ratio of fitness cost and benefit: if the cost is relatively small $(C / B<1 / 3)$, then both autosomal and $X$-linked genes favour exhibiting the ornament (no conflict); if the cost is relatively large $(C / B>1 / 2)$ neither gene favours exhibition of the ornament (no conflict); and if the cost is intermediate $1 / 3<C / B<1 / 2$ ), then the autosoma gene favours and the $\mathrm{X}$-linked gene disfavours exhibition of the ornament (intragenomic conflict).

<Figure B4 here> 
TABLE

\begin{tabular}{|c|c|}
\hline Source & Definition \\
\hline $\begin{array}{l}\text { Cosmides } \\
\text { \& Tooby } \\
{[51]}\end{array}$ & $\begin{array}{l}\text { "The differing inheritance patterns of } \\
\text { cytoplasmic genes and the sex chromosomes } \\
\text { from the Mendelian autosomal patterns can be } \\
\text { used to divide the genome into fractions whose } \\
\text { defining rule is that the fitness of all genes in a } \\
\text { set is maximized in the same way. Each set will } \\
\text { be selected to modify the phenotype of the } \\
\text { organism in a way which maximally propogates } \\
\text { the genes comprising the set, and hence in } \\
\text { ways inconsistent with the other sets which } \\
\text { comprise the total genome. The coexistence of } \\
\text { such multiple sets in the same genome creates } \\
\text { intragenomic conflict" }\end{array}$ \\
\hline
\end{tabular}

Hurst et al. "There is a genetic conflict if the spread of one [33] gene creates the context for the spread of another gene, expressed in the same individual, and having the opposite effect"

Objection

Too restrictive - excludes all intragenomic conflicts arising between genes that share the same mode of transmission, e.g. fair, mendelian, autosomal transmission

Grafen [5] " “...if the p-scores [i.e. the organism's heritable traits] have different maximands, we can ask 'what maximand will the organism appear to be maximizing, if any?'; and we should also expect intraorganismal conflict, as some alleles and traits are selected to oppose the changes that other alleles and traits are selected to promote"

Okasha "The label 'genic selection' will... be reserved [54] for selection between the genes within a single organism or genome, rather than for any selection process that leads to a gene frequency change". "Given this definition, it follows that all outlaws spread by genic selection". "An outlaw, or SGE, is a gene that enjoys a transmission advantage over genes in the same organism but does not increase the organism's fitness... leading to genetic conflict... Such conflicts are usually called 'intra-genomic', for they involve conflict between the different parts of a single genome"

Werren "Genetic conflict occurs when different genetic elements... have influence over the same phenotype, and an increase in transmission of one element by its phenotypic effects causes a decrease in transmission of the other... Genetic conflicts historically have been divided into 'intragenomic' conflict, which occurs within the genome of an individual, and 'intergenomic' conflict, which occurs between individuals... less confusing terms to distinguish these levels may be 'intraindividual' conflict and 'interindividual' conflict, because these terms distinguish genetic conflicts within individual organisms (e.g., for transmission through gametes) as opposed to between individuals

Too persmissive - includes even basic fine-tuning of organismal adaptation

Too restrictive - assumption of fair, mendelian transmission excludes meiotic drive and related conflicts

Too restrictive - excludes all intragenomic conflicts arising from between-organism selection pressures, e.g. parent-of-origin conflicts

Too restrictive - excludes all intragenomic conflicts arising between genes that share the same mode of transmission, e.g. fair, mendelian, autosomal transmission 
(e.g., male-female or parent-offspring conflict over reproductive effort)."

Rice [53] "Genomic conflict occurs when one part of the genome gains a reproductive advantage at the expense of one or more other parts, excluding the intrinsic advantage / expense duality that must occur when one allele is favored over another by simple individual-level selection (selection $_{\text {SIL }}$ ) or the equivalent duality when there is mutualistic coevolution among interacting loci... Genomic parts can be (a) different genetic elements within a single individual... (b) different genes in separate individuals of the same species... or (c) the same genomic region in males and females when there is opposing selection between the sexes"
Too restrictive - excludes all intragenomic conflicts arising between genes that share the same mode of transmission, e.g. fair, mendelian, autosomal transmission

Table 1 | Previous definitions of intragenomic conflict. 


\section{FIGURE LEGEND}

Figure 1 | General classification of intragenomic conflicts. 


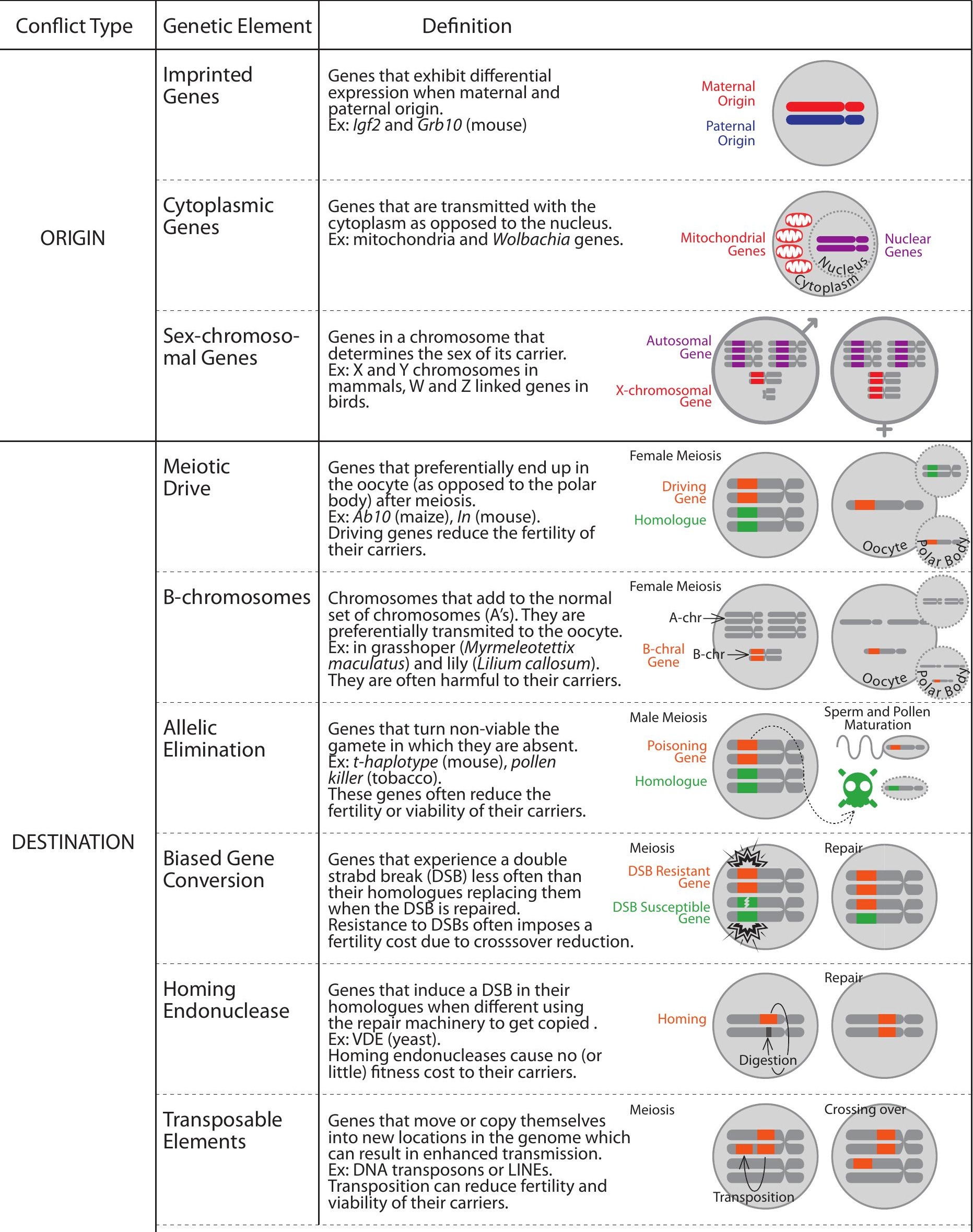

Sex-chromosomal Genes and Cytoplasmic Genes.

\begin{tabular}{l|l} 
Greenbeard & Genes encoding both a cooperative
\end{tabular}

Genes behaviour and causing cooperators to associate (making use of a phenotypic cue -green berad- or by other means). Ex: stla (Photorhabdus), Gp-9 (fire ant)

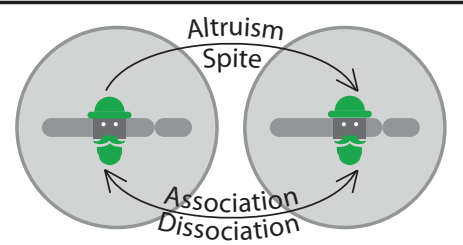



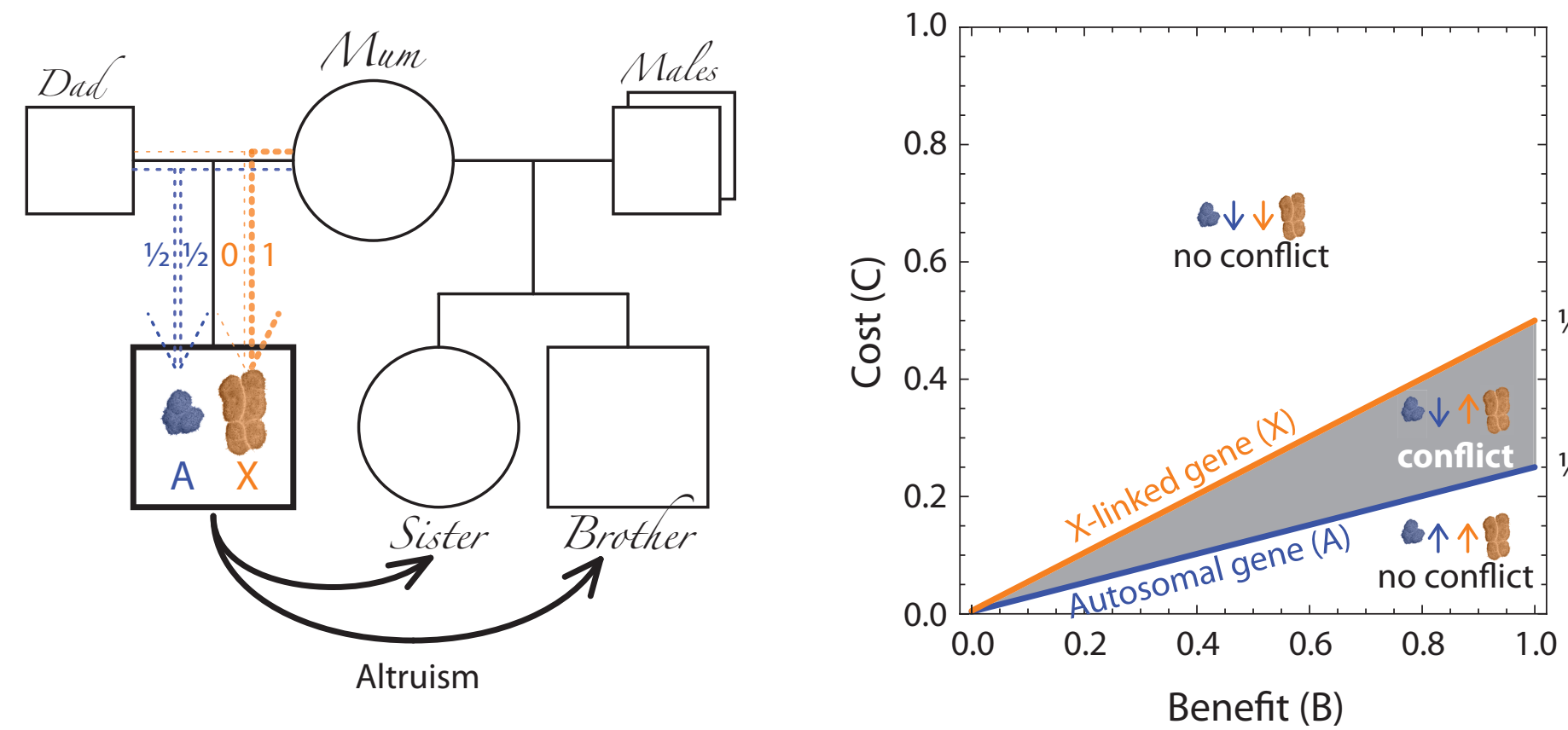

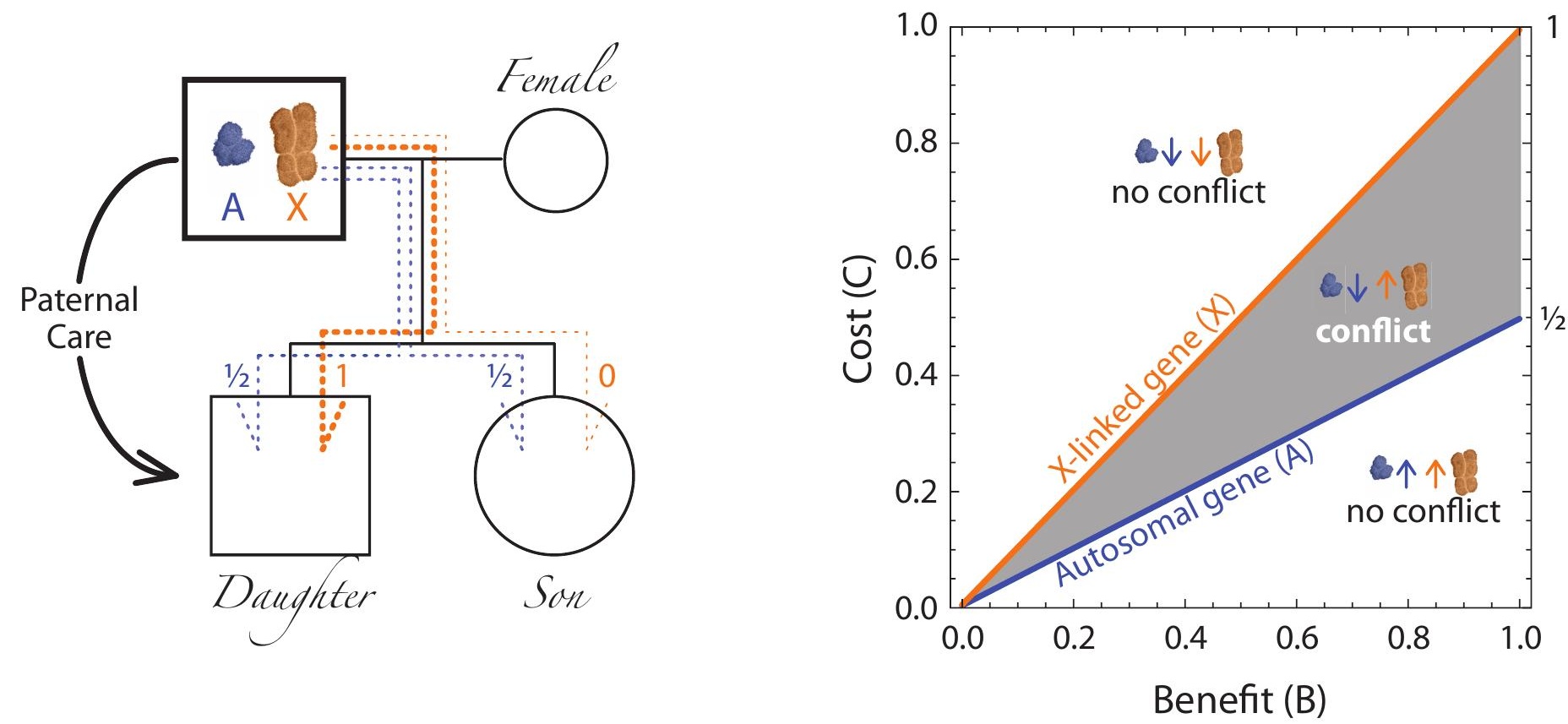

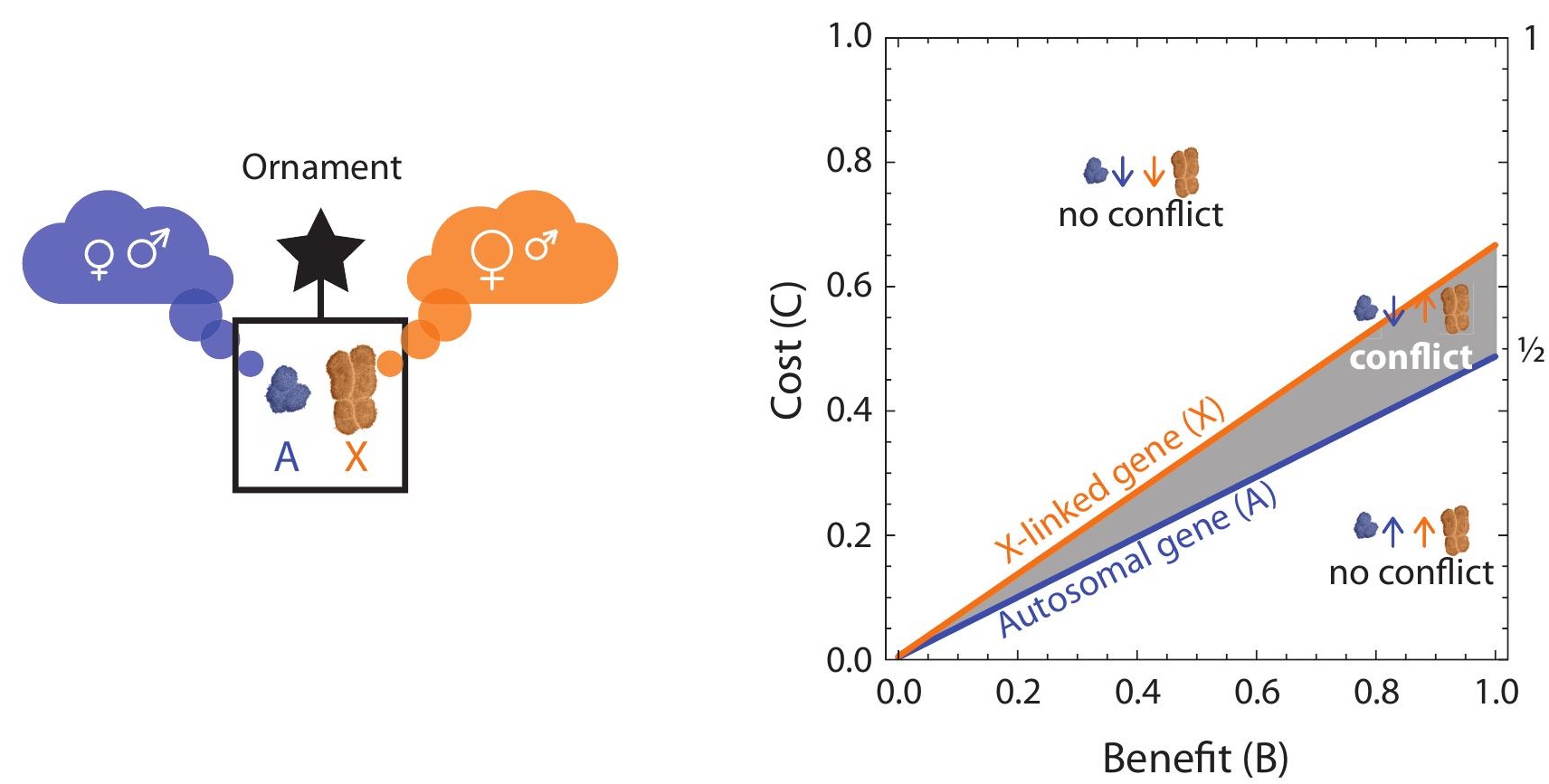